\title{
A novel procedure for Roux-en-Y reconstruction following laparoscopy-assisted distal gastrectomy: transoral placement of anvil and intracorporeal gastrojejunostomy via umbilical mini-laparotomy
}

\author{
Manabu Ohashi · Tomohiro Iwanaga • \\ Ryouki Ohinata - Tatsuo Kanda $\cdot$ Yoshiaki Iwasaki
}

Received: 16 August 2010/ Accepted: 16 November 2010/Published online: 19 February 2011

(c) The International Gastric Cancer Association and The Japanese Gastric Cancer Association 2011

\begin{abstract}
We describe herein our procedures for Rouxen-Y reconstruction using a transoral anvil following laparoscopy-assisted distal gastrectomy (LADG). The procedure consists of three technical processes: transoral placement of the anvil with the head pre-tilted, extracorporeal preparation of the Roux-en-Y limb, and intracorporeal gastrojejunostomy with a circular stapler introduced via an umbilical mini-laparotomy. We applied the procedure to 33 patients with early gastric cancer between December 2008 and June 2010. None of the patients suffered from surgical complications related either to the transoral placement of the anvil or the anastomoses. The postoperative wound appearance was much less conspicuous than that of conventional laparoscopic distal gastrectomy, because our procedure needed only a $4-\mathrm{cm}$ mini-laparotomy on the umbilicus, except for the trocar ports. This technique involving transoral anvil placement enables intracorporeal anastomosis for gastrojejunostomy via an umbilical mini-laparotomy, and may be one of the surgical choices for anastomosis and Roux-en-Y reconstruction following LADG.
\end{abstract}

Keywords Gastric cancer - Laparoscopy-assisted distal gastrectomy · Roux-en-Y reconstruction · Transoral anvil

\footnotetext{
M. Ohashi $(\bowtie) \cdot$ T. Iwanaga $\cdot$ R. Ohinata $\cdot$ Y. Iwasaki Department of Surgery, Tokyo Metropolitan Cancer and Infectious Diseases Center Komagome Hospital, 3-18-22 Honkomagome, Bunkyo-ku, Tokyo 113-8677, Japan e-mail: ohamana@cick.jp

\section{T. Kanda}

Division of Digestive and General Surgery, Niigata University Graduate School of Medical and Dental Sciences, 1-757 Asahimachi-dori, Chuo-ku, Niigata 951-8510, Japan
}

\section{Introduction}

Recent advances in surgical techniques and devices have allowed for adequate regional lymph node dissection, safe reconstruction, and favorable oncologic outcomes in laparoscopy-assisted distal gastrectomy (LADG) for the treatment of gastric cancer [1,2]. However, it remains a challenge how to perform the anastomosis of the gastric remnant safely and steadily, given the limited maneuverability of laparoscopic surgery [3, 4]. Billroth-I reconstruction is commonly selected in LADG mainly because of its ease of performance. Roux-en-Y reconstruction is also selected as an option, especially when a patient has an esophageal hiatus hernia or the gastric remnant becomes too small to enable Billroth-I gastroduodenostomy. Roux-en-Y reconstruction has currently gained favor for anastomosis following LADG, because it is safe and allows for tension-free anastomosis. It also prevents bile reflux into the gastric remnant and the esophagus [5]. However, no standard procedure for Rouxen-Y reconstruction following LADG has been established so far.

Several investigators have advocated diverse procedures for Roux-en-Y reconstruction, using endoscopic linear staplers [4-6] and a circular stapler [7]. Roux-en-Y reconstruction using endoscopic linear staplers enables intracorporeal gastrojejunostomy and allows the completion of not only curative distal gastrectomy but also reconstruction by laparoscopic manipulation [4]. However, this laparoscopic Roux-en-Y reconstruction is relatively complicated and requires expertise as regards intracorporeal stapling techniques. On the other hand, gastrojejunostomy using a circular stapler is simple and readily understandable, but it is used only as an extracorporeal procedure [7]. 
Recently, we designed a new procedure for intracorporeal gastrojejunostomy in Roux-en-Y reconstruction following LADG. In this procedure, we used a transoral anvil to perform the gastrojejunostomy intracorporeally. This anvil was originally developed for use in laparoscopic Roux-en-Y gastric bypass surgery [8] and is characterized by steady and easy placement under conditions of limited manipulability, because the head is pre-tilted and placed transorally. In LADG, the designed procedure enables the creation of an intracorporeal anastomosis between the gastric remnant and the Roux-en-Y limb through a small window that is placed on the umbilicus. We present herein the outline of our procedure and the clinical data of the Roux-en-Y reconstruction using the transoral anvil following LADG.

\section{Patients and methods}

\section{Patients}

Between December 2008 and June 2010, 33 patients with early gastric cancer (29 men and 4 women) underwent Roux-en-Y reconstruction using a transoral anvil following LADG at the Tokyo Metropolitan Cancer and Infectious Diseases Center Komagome Hospital. Median patient age was 64 years (range 33-83 years) and median body mass index (BMI, $\mathrm{kg} / \mathrm{m}^{2}$ ) was 24.1 (range 16.5-35.1). All the patients had histologically proven gastric adenocarcinoma whose depth of invasion was clinically judged as mucosal or submucosal without lymph node metastasis. Before the surgery, written informed consent was obtained from all the patients.

Insertion of gastric tube and mobilization of stomach

Each patient was placed in the supine reverse Trendelenburg position with legs apart, under general anesthesia. Before the start of the operation, an 18-French size gastric tube was transorally introduced into the distal esophagus. A 25-mm transoral anvil (EEA ${ }^{\mathrm{TM}} 25$ Tilt-Top $^{\mathrm{TM}}$ Plus; Covidien, Japan, Tokyo, Japan) was pre-attached to the oral tip of the gastric tube with its head pre-tilted (Fig. 1). After pneumoperitoneum was obtained, six working ports were placed: one at the umbilicus for a laparoscope, one at the epigastrium for a liver retractor, and the remaining four at the bilateral hypochondrium and the mid-abdomen for several kinds of forceps and an energy device. Mobilization of the stomach and lymph node dissection were carried out following a procedure similar to that previously reported by another investigator [1]. The duodenum was divided with the use of a 60-mm endoscopic linear stapler (Endo GIA $^{\mathrm{TM}}$ 60-3.5; Covidien, Japan) after dissection of the infrapyloric nodes.

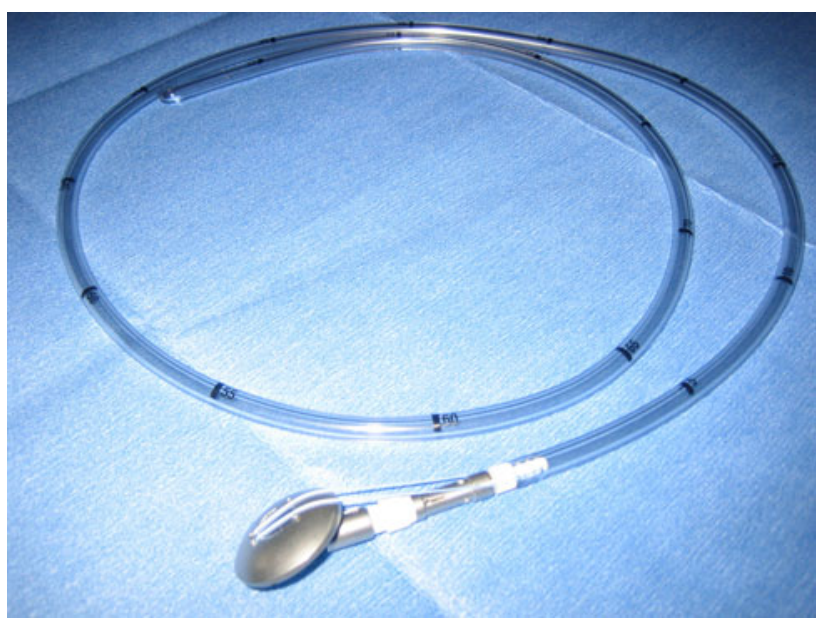

Fig. 1 The transoral anvil used. The head is pre-tilted and attached to the proximal tip of the oral gastric tube

Placement of transoral anvil in gastric remnant

After lymph node dissection of the perigastric and suprapancreatic areas, the stomach was transected using two cartridges of the endoscopic linear stapler. The oral gastric tube to which the anvil was pre-attached was then advanced into the gastric remnant. Once the tip of the oral gastric tube was positioned at the gastric stump, a small gastrotomy was made at the staple line of the greater curvature. The tip of the oral gastric tube was held with grasp forceps and carefully pulled out through the small hole (Fig. 2a). The transoral anvil was then positioned at the cut stump of the gastric remnant. The suture that fixed the anvil to the oral gastric tube was cut. Then, the oral gastric tube was separated from the anvil and removed transabdominally via a $12-\mathrm{mm}$ trocar in the left subcostal area. The anvil was ready for connection to a $25-\mathrm{mm}$ circular stapler $\left(\mathrm{EEA}^{\mathrm{TM}}\right.$ 25-3.5 XL Circular Stapler; Covidien, Japan; Fig. 2b).

Construction of Roux-en-Y limb and preparation for gastrojejunostomy

The umbilical wound was extended to $4 \mathrm{~cm}$ and a protector for the wound (Lap Disk ${ }^{\mathrm{TM}}$; Hakkou, Tokyo, Japan) was inserted to cover the edge of the wound. The surgically excised specimen was taken out of the abdominal cavity and immediately transported to the pathology division.

After pneumoperitoneum was obtained again, the jejunum $20-30 \mathrm{~cm}$ distal from the Treitz ligament was marked as the dividing point to make the Roux-en-Y limb. The jejunum was pulled out of the abdominal cavity via the umbilical mini-laparotomy protected by the Lap Disk ${ }^{\mathrm{TM}}$ and transected on the mark made using an endoscopic 
Fig. 2 The distal tip of the oral gastric tube is held by laparoscopic forceps and the transoral anvil is advanced by pulling out the gastric tube through a laparoscopic port (a). The transoral anvil after removal of the oral gastric tube. The anvil is positioned appropriately at the cut end of the greater curvature (b)
Fig. 3 Appearance after the extracorporeal procedure is completed. a An elastic band is wound around the jejunum to prevent dislocation of the circular stapler. Arrowhead indicates jejunojejunostomy on the Roux-en-Y limb. b The prepared jejunum is returned into the abdominal cavity. The mini-laparotomy is covered with a Lap Disk ${ }^{\mathrm{TM}}$ to establish an airtight condition in the abdominal cavity
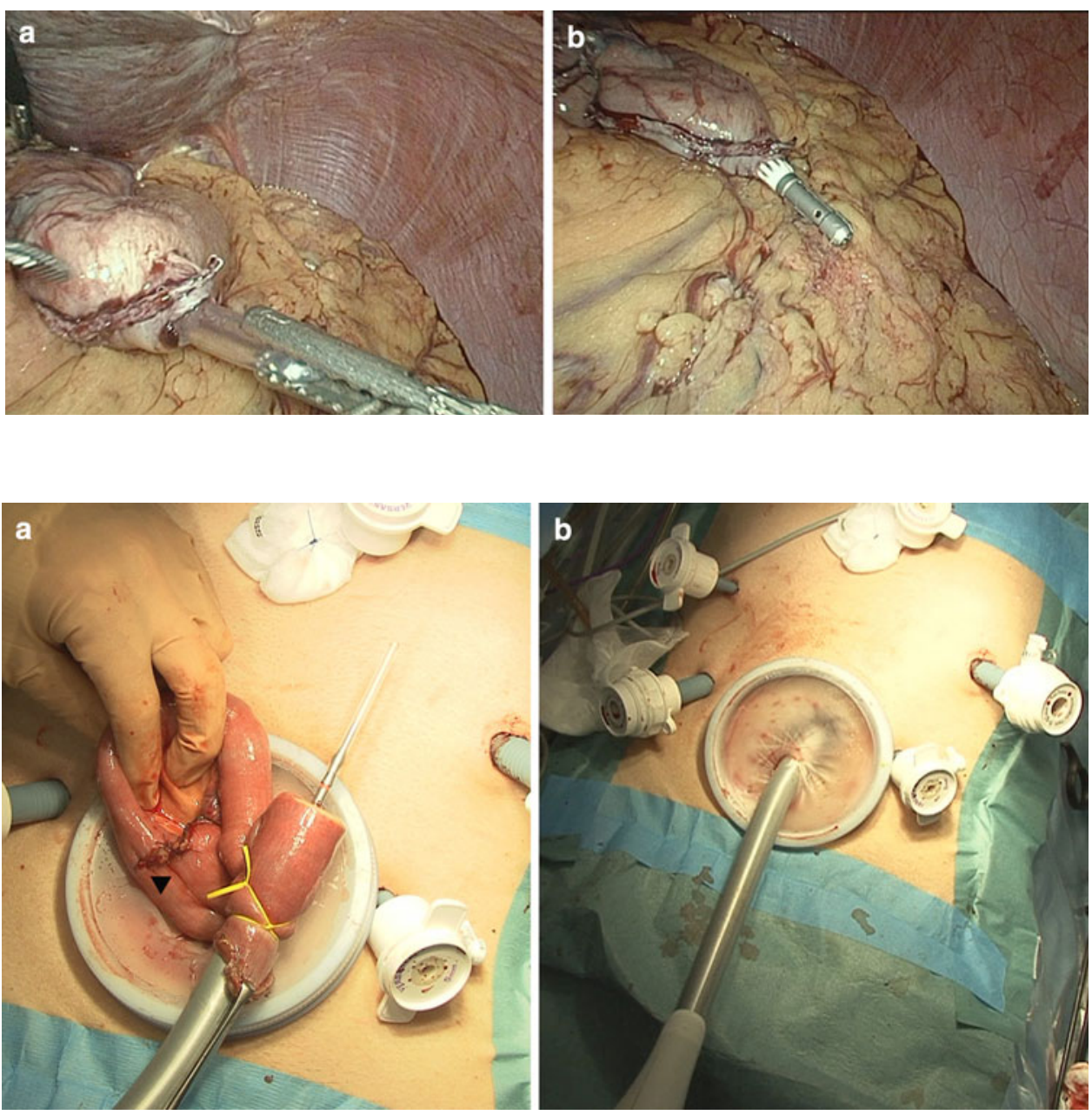

linear stapler. The site of the gastrojejunostomy was determined at the jejunum $10 \mathrm{~cm}$ apart from the cutting edge of the jejunal limb. A jejunojejunostomy was made extracorporeally with an endoscopic linear stapler, in a side-to-side manner, $20 \mathrm{~cm}$ distal from the planned site of the gastrojejunostomy. The common entry hole into which an endoscopic linear stapler was inserted was closed with a continuous single-layer suture.

A circular stapler was introduced into the jejunal limb from the cutting edge and the spike of the circular stapler pierced the jejunal wall at the planned site of the gastrojejunostomy. To prevent dislocation of the circular stapler, an elastic band was wound around the jejunum. Further, the spike of the circular stapler was covered with a silicone tube in order not to injure the adjacent organs during subsequent manipulation (Fig. 3a). The jejunal limb into which the circular stapler was introduced was returned into the abdominal cavity through the Lap Disk ${ }^{\mathrm{TM}}$-covered window (Fig. 3b). The laparoscope was placed through a trocar in the right midabdomen and the laparoscopic procedures were commenced again.
Gastrojejunostomy under laparoscopic view

The extracorporeally prepared jejunal limb was brought up antecolically under laparoscopic view. After removal of the silicone tube, the spike was securely attached to the anvil that was placed in the gastric stump. The pre-tilted anvil automatically returned to the flat position when it was appropriately attached to the body of the circular stapler (Fig. 4a). To keep the lumen of the Roux-en-Y limb, the jejunal limb was released from the elastic tie and pulled laterally to prevent outlet obstruction, which could have been caused by mucosal involvement on firing (Fig. 4b). A blind pouch of the free jejunum was cut using an endoscopic linear stapler (Fig. 4c), completing the gastrojejunostomy in a Roux-en-Y fashion with the transorally placed anvil (Fig. 4d).

\section{Postoperative management}

All patients were cared for according to the predetermined schedule, named the "clinical pathway for LADG" in our hospital: water intake was begun on postoperative day (POD) 2 and oral feeding, on POD 4. Discharge was 
Fig. 4 The pre-tilted anvil head automatically returns to the flat position when the spike of the circular stapler is properly attached to the anvil (a). Firing of the cartridge is carried out under laparoscopic observation. To prevent entanglement of the mesenteric side of the jejunum, the opposite side of the mesentery is manipulated to form a right angle between the circular stapler and the efferent jejunum (b). The jejunal stump is closed with an endoscopic linear stapler (c). Appearance after the intracorporeal gastrojejunostomy using the transoral anvil is completed (d)
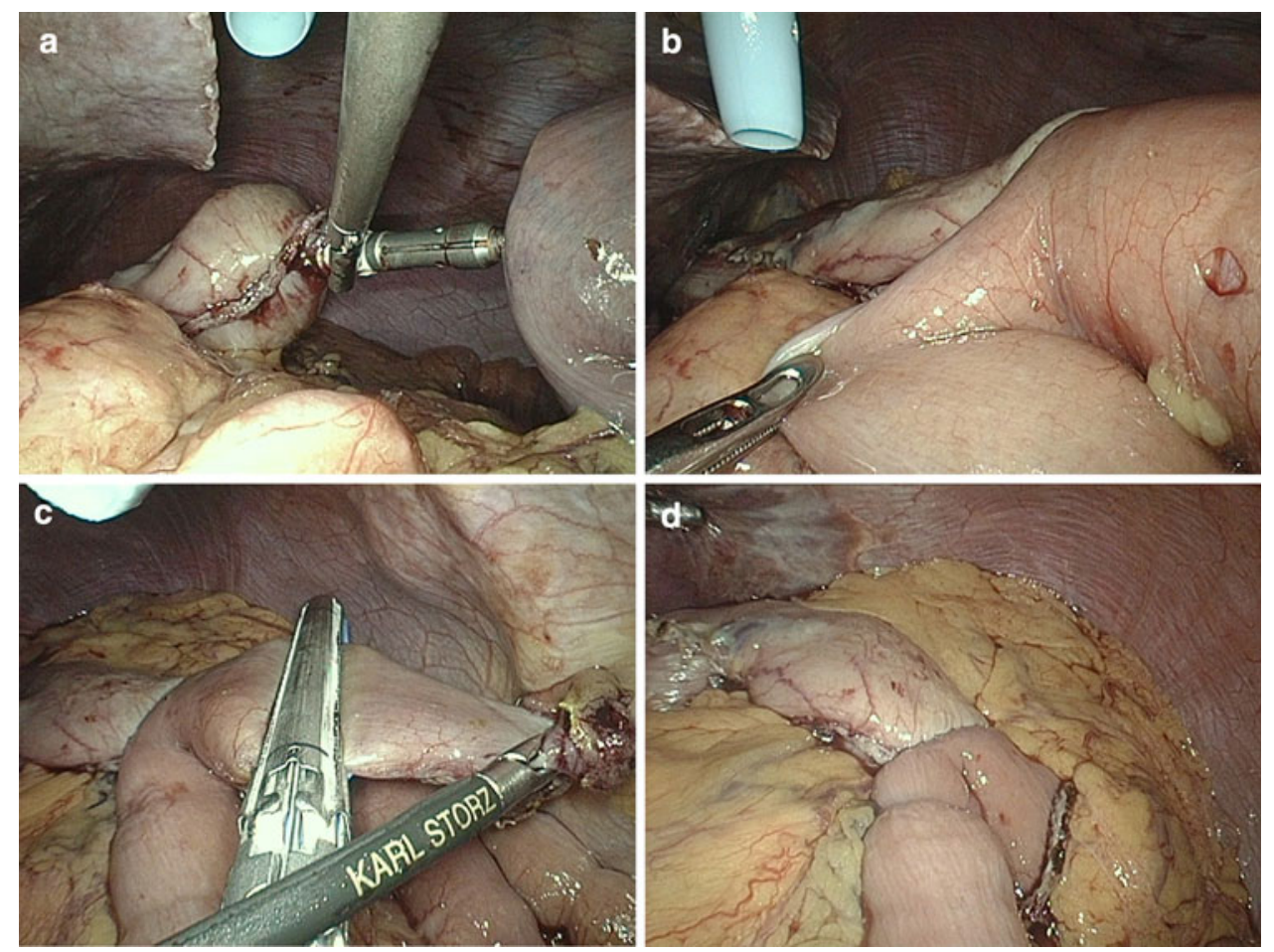

allowed if the patients could have solid meals without any troubles on POD 8.

\section{Results}

Intraoperative safety

In all the 33 patients enrolled, the Roux-en-Y gastrojejunostomy using the transoral anvil was accomplished with no major complications. In one patient, the transoral anvil was stuck at entry into the cervical esophagus when the gastric tube was pulled out for the anvil placement, and the anvil had to be manually removed from the mouth. In this patient, the anvil was reintroduced transorally and successful entry into the esophagus was achieved by lifting the larynx extracorporeally. None of the patients suffered from sore throat or other injuries of the esophagus.

There were no complications during the extracorporeal procedures for the construction of the Roux-en-Y limb and the introduction of the stapler shaft into the jejunum. Furthermore, no difficulty was noted in docking the spike of the circular stapler to the transoral anvil or in firing under laparoscopic view.

\section{Operation time}

The median operation time of the 33 patients was $272 \mathrm{~min}$ (range 173-352 $\mathrm{min}$ ). Analyzing the operation time based on operation date, the median operation time of the 16 patients in the earlier half of the study period was $281 \mathrm{~min}$ (range 209-322 $\mathrm{min}$ ) and that of the 17 patients in the later half was $257 \mathrm{~min}$ (range 173-352 $\mathrm{min}$ ).

Postoperative outcomes

No postoperative bleeding, leakage, or stricture of the gastrojejunostomy was noted in this series. Two of the 33 patients were rehospitalized because of insufficient food intake, probably caused by Roux stasis syndrome. Both patients recovered non-surgically within several weeks.

\section{Discussion}

We designed a procedure for Roux-en-Y reconstruction following LADG, in which we employed a newly developed transoral anvil placement technique. Our procedure consists of three technical processes: (1) transoral placement of the anvil, (2) extracorporeal manipulations consisting of jejunojejunostomy and setting the stapler at the jejunal end, and (3) intracorporeal manipulations consisting of gastrojejunostomy with a circular stapler introduced via an umbilical mini-laparotomy and closing the jejunal end with a linear stapler. The results obtained from our initial series of patients showed the feasibility of this technique and demonstrated the safety of the anastomosis. To the best of our knowledge, this is the first report of the use of a 
transoral anvil placement technique in Roux-en-Y reconstruction following LADG.

The present Roux-en-Y reconstruction is essentially a modification of the procedure initially reported by Toyama et al. [7] in which a circular stapler is used for gastrojejunostomy following LADG. In the present procedure, the process involved in the appropriate transoral placement of the anvil in the gastric stump by laparoscopic manipulation received the greatest attention. In 2000 when the transoral anvil was still not available commercially, Nguyen and Wolfe [8] reported a patient with morbid obesity with hypopharyngeal perforation after the attempted transoral insertion of an ordinary anvil, without the head pre-tilted, for laparoscopic gastric bypass. However, Nguyen et al. [9] reported later that when they used a transoral anvil, the pre-tilted anvil head markedly reduced resistance in passage, and successful placement was realized in 23 patients with morbid obesity undergoing laparoscopic gastric bypass. Sakuramoto et al. [10] reported the results of using the transoral anvil placement technique for laparoscopic esophagojejunostomy following total gastrectomy. Of the 27 patients in their series, the anvil placement failed in only one patient, who had esophageal stricture. Both the above groups reported no complications related to the transoral anvil placement, revealing the safety of this technique. In the 33 patients in our series, transoral anvil placement was achieved in all without inducing complications associated with this procedure. Based on these results, we can conclude that the transoral placement of the pre-tilted anvil is a safe and reliable technique that is suitable for surgeries where manipulability is limited, such as laparoscopic bariatric surgery and gastrectomy, unless there is an obstacle between the oral cavity and the gastric remnant.

When the procedure using the transoral anvil with a circular stapler for reconstruction following LADG was first introduced, we were worried that gastrojejunostomy using a circular stapler would increase the incidence of anastomotic bleeding and stricture because of the abundant blood circulation in the cutting edge of the gastric wall and the excessive tissue proliferation promoted by the metallic staplers. However, in the present series, none of the 33 patients suffered from anastomotic bleeding or stricture. Hori et al. [11] reported the results of a prospective randomized trial of hand-sutured versus mechanically stapled anastomoses for gastroduodenostomy after distal gastrectomy. They described that of the 92 patients undergoing gastrojejunostomy using a circular stapler, one $(1.1 \%)$ suffered from anastomotic bleeding and four $(4.3 \%)$, suffered from stricture, and the incidence of the anastomotic complications was comparable to that of hand-sutured gastroduodenostomy. Yang et al.
[12] reported that the incidence of anastomotic complications, including bleeding, stricture, and leakage, in 440 hemi-double stapling gastroduodenostomies using a circular stapler was $1.2 \%$. In Roux-en-Y gastrojejunostomy using a $25-\mathrm{mm}$ circular stapler in bariatric surgery, Suggs et al. [13] detected anastomotic bleeding and stricture in 1.6 and $2.9 \%$, respectively, of 374 patients with morbid obesity. These studies underpin the fact that gastroenterostomy using a circular stapler does not increase the incidence of anastomotic complications. However, as the incidence of anastomotic complications is quite low, further accumulation of clinical data is required to clarify the anastomotic outcomes of gastrojejunostomy using the transoral anvil with a circular stapler under laparoscopic view.

In our Roux-en-Y reconstruction, preparation of the Roux-en-Y limb and jejunojejunostomy are conducted extracorporeally. These procedures can be conducted through a small window, as the jejunum is an organ with high mobility. The wounds necessitated by this surgery are not different from those necessitated by totally laparoscopic distal gastrectomy (TLDG), which is completed with laparoscopic maneuvers alone. Thus, our procedure provides the same cosmetic benefits to patients as TLDG. TLDG is one of the optimal goals of laparoscopic surgery for gastric cancer because it eliminates laparotomy at the upper abdomen, thereby maximizing the cosmetic benefits and reducing the invasiveness that conventional laparoscopic surgery potentially creates. To achieve TLDG, Kanaya et al. [3] developed delta anastomosis for Billroth-I reconstruction, and Takaori et al. [4] first reported intracorporeal functional end-to-end gastrojejunostomy and jejunojejunostomy for Roux-en-Y reconstruction. Despite the excellent cosmetic benefits, the techniques of their laparoscopic anastomoses were quite complicated and specialized for laparoscopic experts alone. Our present procedure is different from TLDG, as the jejunal anastomosis and the setting of the circular stapler are conducted extracorporeally. However, our procedure requires only an umbilical mini-laparotomy $4 \mathrm{~cm}$ in length from which surgical specimens are removed and the jejunum is pulled out for preparation of the Roux-enY limb and jejunojejunostomy. Moreover, the operation time of our procedure is not prolonged; it is similar to that of conventional LADG with Roux-en-Y reconstruction using linear staplers reported by Kojima et al. [5]. We are convinced that our procedure can afford the same advantages as TLDG in terms of low invasiveness and postoperative cosmetic appearance.

One disadvantage of our procedure is the cost, because a large number of suture devices are needed. In this procedure, we used six cartridges of an endoscopic linear stapler, one transoral anvil, and one circular stapler. On the other 
hand, the previously reported TLDG procedures [4-6] required six or seven cartridges of an endoscopic linear stapler alone. To circumvent the disadvantage in cost, jejunojejunostomy on the Roux-en-Y limb is currently made by hand suture, which could spare the use of two cartridges of the endoscopic linear stapler.

In conclusion, Roux-en-Y gastrojejunostomy using the transoral anvil is a safe and convenient technique for LADG. Our device facilitates the reconstruction of a complicated gastrojejunostomy in a Roux-en-Y fashion following LADG and has the same advantage as TLDG in terms of postoperative cosmetic appearance. This procedure is expected to be useful for reconstruction following LADG, although its safety and benefits should be further clarified in future studies.

\section{References}

1. Noshiro H, Nagai E, Shimizu S, Uchiyama A, Tanaka M. Laparoscopically assisted distal gastrectomy with standard radical lymph node dissection for gastric cancer. Surg Endosc. 2005;19:1592-6.

2. Kitano S, Shiraishi N, Uyama I, Sugihara K, Tanigawa N, Japanese Laparoscopic Surgery Study Group. A multicenter study on oncologic outcome of laparoscopic gastrectomy for early cancer in Japan. Ann Surg. 2007;245:68-72.

3. Kanaya S, Gomi T, Momoi H, Tamaki N, Isobe H, Katayama T, et al. Delta-stapling anastomosis in totally laparoscopic Billroth I gastrectomy: new technique of intraabdominal gastrojejunostomy. J Am Coll Surg. 2002;195:284-7.
4. Takaori K, Nomura E, Mabuchi H, Lee SW, Agui T, Miyamoto $\mathrm{Y}$, Iwamoto $\mathrm{M}$, Watanabe $\mathrm{H}$, Tanigawa $\mathrm{N}$. A secure technique of intracorporeal Roux-Y reconstruction after laparoscopic distal gastrectomy. Am J Surg. 2005;189:178-83.

5. Kojima K, Yamada H, Inokuchi M, Kawano T, Sugihara K. A comparison of Roux-en-Y and Billroth-I reconstruction after laparoscopy-assisted distal gastrectomy. Ann Surg. 2008;247: 962-7.

6. Noshiro H, Ohuchida K, Kawamoto M, Ishikawa M, Uchiyama A, Shimizu S, Tanaka M. Intraabdominal Roux-en-Y reconstruction with a novel stapling technique after laparoscopic distal gastrectomy. Gastric Cancer. 2009;12:164-9.

7. Toyama E, Honda S, Baba Y, Ishikawa S, Hayashi N, Miyanari N, Baba H. "Tornade Roux-en-Y" anastomosis in laparoscopyassisted distal gastrectomy. Gastric Cancer. 2008;11:181-5.

8. Nguyen NT, Wolfe BM. Hypopharyngeal perforation during laparoscopic Roux-en-Y gastric bypass. Obes Surg. 2000;10: 64-7.

9. Nguyen NT, Hinojosa MW, Smith BR, Reavis KM, Wilson SE. Advances in circular stapling technique for gastric bypass: transoral placement of the anvil. Obes Surg. 2008;18:611-4.

10. Sakuramoto S, Kikuchi S, Futawatari N, Moriya H, Katada N, Yamashita K, et al. Technique of esophagojejunostomy using transoral placement of the pretilted anvil head after laparoscopic gastrectomy for gastric cancer. Surgery. 2010;147:742-7.

11. Hori S, Ochiai T, Gunji Y, Hayashi H, Suzuki T. A prospective randomized trial of hand-sutured versus mechanically stapled anastomoses for gastroduodenostomy after distal gastrectomy. Gastric Cancer. 2004;7:24-30.

12. Yang HK, Lee HJ, Ahn HS, Yoo MW, Lee IK, Lee KU. Safety of modified double-stapling end-to-end gastroduodenostomy in distal subtotal gastrectomy. J Surg Oncol. 2007;96:624-9.

13. Suggs WJ, Kouli W, Lupovici M, Chau WY, Brolin RE. Complications at gastrojejunostomy after laparoscopic Roux-en-Y gastric bypass: comparison between 21- and 25-mm circular staplers. Surg Obes Relat Dis. 2007;3:508-14. 